\title{
Design and Analysis of a Simple Nonlinear Vibration Absorber
}

\author{
Abdullah S. Alsuwaiyan \\ Mechanical Engineering Department-Unayzah College of Engineering - Qassim University- Saudi Arabia
}

\begin{abstract}
In this work, a system consisting of a main structure with a nonlinear tuned vibration absorber having a hardening spring attached to it is considered. The nonlinearity in the absorber's spring is a cubic nonlinearity and is obtained by attaching two additional, relatively soft, similar linear springs to the absorber's mass. These added springs are attached to the absorber's mass in such a way that they are initially normal to the absorber's displacement axis. The dynamics of main structure and that of the nonlinear absorber are analyzed using the first order harmonic balancing method (HB1) and the results are compared against those of a system composing of a linear vibration absorber attached to a similar main structure. A numerical example is considered. The results obtained are in agreement with the known features of nonlinear absorbers. They showed that the absorber's effective frequency band width is increased when a nonlinear absorber is employed instead of a linear one. The results also showed that the displacement amplitude of the absorber during operation is decreased when using a nonlinear absorber. It is also found that the absorber's effective band width depends on the original length of the added linear springs, that are used to obtain the nonlinearity in the absorber, in such a manner that it increases as the original length of the two springs is decreased.
\end{abstract}

Keywords: tuned absorber, vibration absorber, nonlinear absorber, band width, harmonic balancing

\section{Introduction}

Hermann Frahm[1] invented Dynamic Vibration Absorber (DVA) in 1909 and its characteristics were studied in depth by Den Hartog [2] in 1959. Since then, DVA became of interest to many researchers and has been extensively used in eliminating or reducing unwanted vibrations in many engineering applications. It is practically the most popular passive device for vibration mitigation. Although there are other ways to reduce unwanted vibrations, the DVA has certain advantages over other methods. In its simplest form, DVA consists of a mass attached by an elastic element to the primary structure whose vibration is to be suppressed. Being external to the primary structure, so no re-installation of equipment is necessary, is one of the big advantages of DVA. Another big advantage is that it can be designed and tested before being installed. It can be adjusted in the laboratory environment with predictable field results.

The DVA mass and spring are chosen such that they will resonate at a particular frequency called the tuned frequency. Ideally, with the addition of the tuned DVA, the main mass will not vibrate at that frequency regardless of the excitation amplitude applied. However, the perfectly tuned DVA has very little effect on all but the closest frequencies to the tuned frequency. Thus, tuned DVAs are generally only effective if the principle frequency of excitation is well known and constant. As the excitation frequency drifts from the tuned frequency, tuned DVA becomes less effective. In other words, the principle drawback of tuned DVA is its small effective frequency band width.

The tuned DVA's important limiting feature of having small effective band width directed researches toward searching for modifications and improvements to overcome this drawback. Some researchers considered multiple absorbers to overcome this disadvantage, see for example $[3,4,5,6]$ and the references therein. Although linear vibration absorbers were and still are of interest to many researchers, many other researchers considered implementing nonlinear absorbers to overcome the above mentioned drawback of tuned DVA. One of the earliest studies on nonlinear vibration absorbers was the work by Roberson [7]. He investigated the performance of an undamped nonlinear vibration absorber and concluded that the absorbers' effective band width improves significantly when a nonlinear absorber is used instead of a linear one. Many other researchers then became interested in nonlinear absorbers. For example, a nonlinear absorber with a softening spring consisting of a stack of Belleville washers was considered by Hunt and Nissen [8] to overcome the previously mentioned drawback of linear absorbers. Nissen et al. [9] studied the optimal parameters of a nonlinear absorber and considered the technical aspects for realization. Optimal parameter design of linear and nonlinear dynamic vibration absorbers for damped primary systems were investigated by Soom [10] and Jordanov [11]. They investigated optimization criteria other than traditional measures and obtained a small improvement in the steady state response by using nonlinear springs.

Other researchers, however, pointed out that there are some unpleasant features of the nonlinear absorbers. For example, Rice [12] and Shaw et al. [13] studied the response of the nonlinear vibration absorber and pointed out that nonlinearities introduce dangerous instabilities that may increase unwanted vibration in the system instead of suppressing it. 
Studies on linear and nonlinear absorbers are still going on. This work is aimed to be a contribution in this direction. Here, a simple spring configuration that gives a spring element with a cubic nonlinear stiffness is considered. It is installed in a vibration absorber attached to a primary mass under single harmonic excitation. The system is then analyzed analytically using the harmonic balancing method and is compared with the tuned linear DVA.

\section{Mathematical Background}

A primary structure attached to ground by a linear spring and a viscous damper and subjected to a single harmonic excitation force with a vibration absorber attached to it as shown schematically in Fig. 1 is considered. The equations of motion that describe the dynamics of this system are:

$$
\begin{gathered}
m \ddot{x}+c \dot{x}+k x-c_{a}\left(\dot{x}_{a}-\dot{x}\right)-k_{a}\left(x_{a}-x\right)-\alpha_{a}\left(x_{a}-x\right)^{3}=F_{o} \sin \omega t \\
m_{a} \ddot{x}_{a}+c_{a}\left(\dot{x}_{a}-\dot{x}\right)+k_{a}\left(x_{a}-x\right)+\alpha_{a}\left(x_{a}-x\right)^{3}=0
\end{gathered}
$$

where, $\left(^{\cdot}\right)=\frac{d}{d t}(\quad),\left(^{*}\right)=\frac{d^{2}}{d t^{2}}(\quad), m, c, k$ are the main structure mass, its damping coefficient and its spring stiffness respectively and $m_{a}, c_{a}, k_{a}$ are those for the absorber. The coordinates $x$ and $x_{a}$ are the displacement coordinates of the main mass and the absorber mass respectively. Lastly, $\alpha_{a}$ is the coefficient of the cubic nonlinearity in the absorber spring.

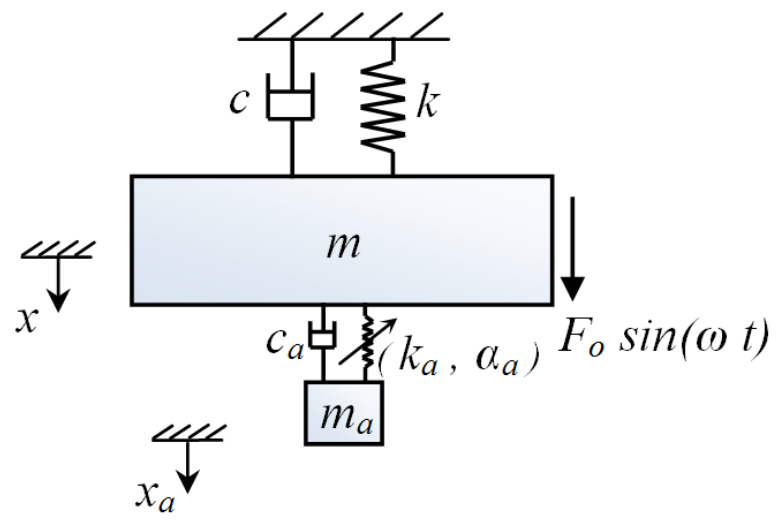

Fig. 1. Schematic diagram of a main mass with a DVA

The steady state amplitudes of vibration of the main structure and the absorber can be found by solving these equations. To do so, we will consider two cases, namely linear vibration absorber case and nonlinear vibration absorber case.

\section{LINEAR VIBRATION ABSORBER} matrix form:

For the case of a linear absorber, i.e., $\alpha_{a}=0$, equations (1) and (2) can be written in the following

$$
\boldsymbol{M} \ddot{\boldsymbol{x}}+\boldsymbol{C} \dot{\boldsymbol{x}}+\boldsymbol{K} \boldsymbol{x}=\boldsymbol{F} \sin (\omega t)
$$

where, the vectors $\boldsymbol{x}$ and $\boldsymbol{F}$ are:

and the matrices $\boldsymbol{M}, \boldsymbol{C}$, and $\boldsymbol{K}$ are:

$$
\boldsymbol{x}=\left(\begin{array}{c}
x \\
x_{a}
\end{array}\right), \quad \boldsymbol{F}=\left(\begin{array}{c}
F_{o} \\
0
\end{array}\right)
$$

$$
\boldsymbol{M}=\left(\begin{array}{cc}
m & 0 \\
0 & m_{a}
\end{array}\right), \quad \boldsymbol{C}=\left(\begin{array}{cc}
c+c_{a} & -c_{a} \\
-c_{a} & c_{a}
\end{array}\right), \quad \boldsymbol{K}=\left(\begin{array}{cc}
k+k_{a} & -k_{a} \\
-k_{a} & k_{a}
\end{array}\right)
$$

The steady state response of the main mass and the absorber mass will be of the form:

$$
\boldsymbol{x}=\left[\begin{array}{c}
X \sin \left(\omega t+\phi_{1}\right) \\
X_{a} \sin \left(\omega t+\phi_{2}\right)
\end{array}\right]=\boldsymbol{A} \sin (\omega t)+\boldsymbol{B} \cos (\omega t)
$$

where, $\boldsymbol{A}=\left(\begin{array}{l}a_{1} \\ a_{2}\end{array}\right), \boldsymbol{B}=\left(\begin{array}{l}b_{1} \\ b_{2}\end{array}\right)$

The main mass and the absorber mass vibration amplitudes from equation (4) are:

$$
X_{1}=\sqrt{a_{1}^{2}+b_{1}^{2}}, \quad X_{a}=\sqrt{a_{2}^{2}+b_{2}^{2}}
$$


Appling simple linear algebra, one can reach to the following expressions for the vectors A and B:

$$
\left(\begin{array}{l}
\boldsymbol{A} \\
\boldsymbol{B}
\end{array}\right)_{4 \times 2}=\left[\begin{array}{cc}
\boldsymbol{K}-\omega^{2} \boldsymbol{M} & -\omega \boldsymbol{C} \\
\omega \boldsymbol{C} & \boldsymbol{K}-\omega^{2} \boldsymbol{M}
\end{array}\right]_{4 \times 4}^{-1}\left(\begin{array}{c}
\boldsymbol{F} \\
\mathbf{0}
\end{array}\right)_{4 \times 2}
$$

\section{NONLINEAR VIBRATION ABSORBER}

Here a cubic nonlinearity in the absorber spring is allowed, i.e., $\alpha_{a} \neq 0$. A simple way to obtain such a spring is by employing the spring configuring shown in Fig. 2. To show that this simple configuration gives the desired stiffness, suppose the point that joins the two springs is displaced downward by a displacement $y$, the restoring force in the $y$ direction, $F_{s v}$, coming from the two springs will then be:

$$
F_{s v}=2 \frac{y}{\sqrt{L^{2}+y^{2}}}\left[k_{h}\left(\sqrt{L^{2}+y^{2}}-L\right)+F_{s o}\right]
$$

where, $F_{s o}$ is the initial force in each spring. Expanding this in power series about $\mathrm{y}=0$, yields:

$$
F_{S v}=\frac{2 F_{S O}}{L} y+\left(\frac{k_{h} L-F_{S o}}{L^{3}}\right) y^{3}+O\left(y^{5}\right)
$$

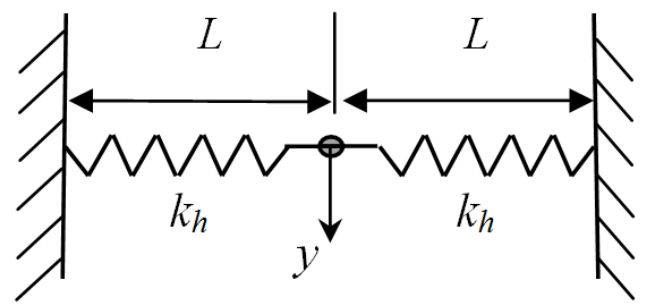

Fig. 2. Springs configuration to obtain a combined linear and cubic nonlinear stiffness in the vertical direction

It is clear from equation (8) that one can obtain the desired spring effect just by choosing the parameters $F_{s o}, k$, and $L$. Fig. 3 schematically shows this spring configuration employed in the dynamic vibration absorber.

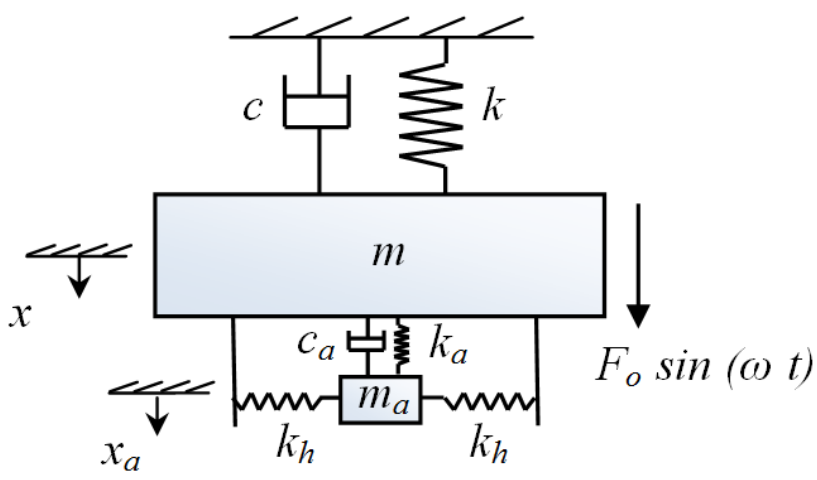

Fig. 3. A schematic diagram of the main mass and an absorber with springs configuration giving linear and cubic nonlinear spring stiffness effect

To find the system response, equations (1) and (2) need to be solved. To do so, first order harmonic balancing method, HB1, is employed but first it is more convenient to rewrite equations (1) and (2) in nondimensional forms as follows:

$$
\begin{gathered}
x_{n}^{\prime \prime}+\frac{2}{(1+\mu)} \xi x_{n}^{\prime}+x-\frac{\mu}{(1+\mu)}=\frac{1}{(1+\mu)} \sin (r \tau) \\
z_{n}^{\prime \prime}+2 \xi_{a} r_{o} z_{n}^{\prime}+r_{o}^{2} z+\gamma z^{3}=x_{n}^{\prime \prime}
\end{gathered}
$$

where, 


$$
\begin{aligned}
& \tau=\omega_{1} t,()^{\prime}=\frac{d}{d \tau}(),()^{\prime \prime}=\frac{d^{2}}{d \tau^{2}}(), x_{n}=x \frac{k}{F_{o}}, z_{n}=\frac{\left(x-x_{a}\right) k}{F_{o}}, \mu=\frac{m_{a}}{m}, \\
& \xi=\frac{c}{2 \sqrt{k m}}, \xi_{a}=\frac{c_{a}}{2 \sqrt{k_{a} m_{a}}}, r_{o}=\frac{\omega_{a}}{\omega_{1}}, r=\frac{\omega}{\omega_{1}}, \omega_{a}=\sqrt{\frac{k_{a}}{m_{a}}}, \omega_{1}=\sqrt{\frac{k}{m}}, \gamma=\frac{\alpha F_{o}^{2}}{\mu k^{3}}
\end{aligned}
$$

Many researchers implemented harmonic balancing method in studying nonlinear dynamics applications, see for example [14-15] and the references therein. In implementing the first order harmonic balance method, it is assumed that the displacement of the main mass and that of the absorber will be harmonic with both having a frequency equal to the applied force frequency, or in equation form:

$$
x_{n}=X_{n} \sin (r \tau+\phi), z_{n}=Z_{n} \sin (r \tau+\theta)
$$

To find the amplitudes of vibration, $X_{n}$ and $Z_{n}$, equations (11) need to be substituted in equations (9) and (10), then collect the coefficients of similar trigonometric functions to form a set of nonlinear algebraic equations. The solutions of the resulting algebraic equations give the desired amplitudes of vibration. Doing that, the following expressions for the amplitudes of vibrations can be reached [16]:

$$
\begin{aligned}
& X_{n}=\frac{1}{r^{2}} \sqrt{a Z_{n}^{6}+b Z_{n}^{4}+d Z_{n}^{2}} \\
& a Z_{n}^{6}+e Z_{n}^{4}+f Z_{n}^{2}+g=0
\end{aligned}
$$

where,

$$
\begin{aligned}
& a=\frac{9}{16} \gamma^{2}, \quad b=\frac{3}{2} \gamma\left(r_{o}^{2}-r^{2}\right), d=r^{4}+4 \xi_{a}^{2} r^{2} r_{o}^{2}+r_{o}^{4}-2 r^{2} r_{o}^{2}, \quad e=b+r^{4} \frac{h}{p} \\
& f=\left(r_{o}^{2}-r^{2}\right)^{2}+4 \xi_{a}^{2} r^{2} r_{o}^{2}+r^{4} \frac{q}{p}, g=-\frac{r^{4}}{p}, h=-\frac{3}{2} \gamma \mu\left(1-r^{2}(1+\mu)\right), \\
& p=\left(1-r^{2}(1+\mu)\right)^{2}+4 \xi^{2} r^{2}, \quad q=r^{4} \mu^{2}-2 \mu\left(1-r^{2}(1+\mu)\right)\left(r_{o}^{2}-r^{2}\right)+8 \xi \xi_{a} \mu r_{o} r^{2}
\end{aligned}
$$

Equation (13) is solved for the relative vibration amplitude of the absorber. The solutions of (13) are:

$$
\begin{gathered}
Z_{n}^{1}=\sqrt{w+n-\frac{e}{3 a}} \\
Z_{n}^{2,3}=\sqrt{-\frac{1}{2}(w+n)-\frac{e}{3 a} \pm j \frac{\sqrt{3}}{2}(w-n)}
\end{gathered}
$$

Where,

$$
\begin{aligned}
& w=\left(v+\sqrt{-\frac{\kappa}{108 a^{4}}}\right)^{\frac{1}{3}}, n=\left(v-\sqrt{-\frac{\kappa}{108 a^{4}}}\right)^{\frac{1}{3}}, v=\frac{9 a e f-27 a^{2} g-2 e^{3}}{54 a^{3}}, \\
& \kappa=18 \text { aef } g-27 a^{2} g^{2}-4 e^{3} g+e^{2} f-4 f^{3} a
\end{aligned}
$$

The absorber normalized relative amplitudes of vibration from equations (14) and (15) are then substituted in equation (12) to find the main mass normalized amplitudes of vibration. It should be noted here that only real roots are accepted since complex roots don't have physical meanings.

\section{Numerical Example and Discussion}

As an example, a vibration absorber for suppressing the excessive vibration of a machine over the widest possible frequency range of the single harmonic excitation force applied on the machine and causing it to vibrate is to be designed and analyzed. The magnitude of the excitation force, $F_{o}$, is taken to be $1 \mathrm{kN}$. The machine is modeled as a simple mass of $100 \mathrm{~kg}$ with light damping and has a natural frequency of $15 \mathrm{rad} / \mathrm{s}$. The mass of the absorber, $m_{a}$, and its linear spring stiffness, $k_{a}$, are chosen so that the primary resonance of the main system and its damaging effects are avoided. The numerical values of the absorber mass and its linear stiffness are $10 \mathrm{~kg}$ and $2250 \mathrm{~N} / \mathrm{m}$.

Fig. 4 shows the normalized amplitude of vibration of the machine with and without attaching the linear vibration absorber and for different frequency ratios. It is clear that the linear absorber is effectively doing its job in suppressing the main mass vibration at an excitation frequency equal to the natural frequency of the main mass and at the very close nearby frequencies. 


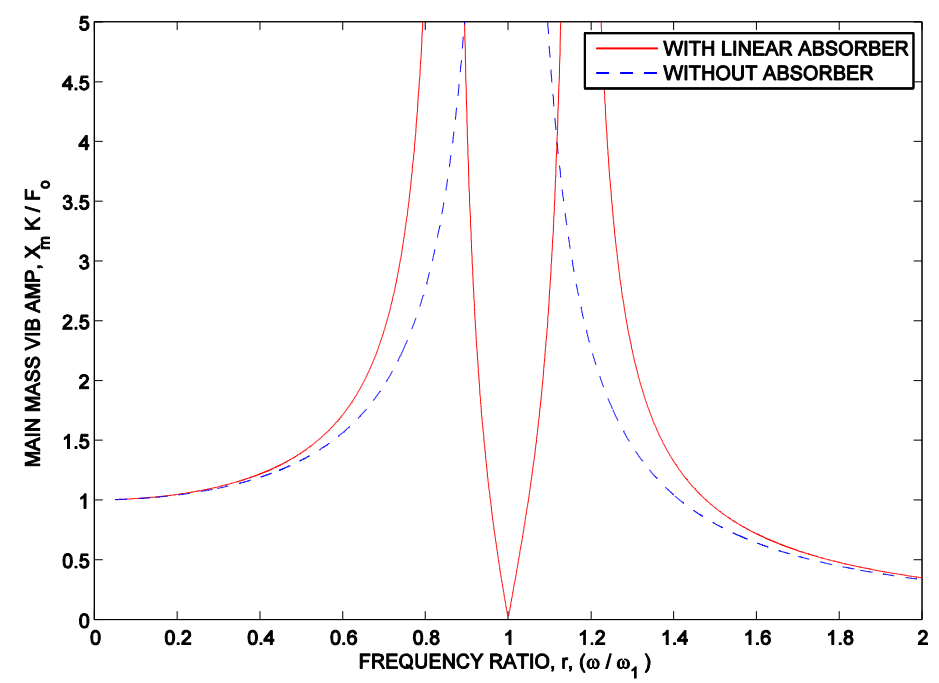

Fig. 4. Normalized amplitude of vibration for the main mass for different frequency ratios

Now, as pointed out earlier, a nonlinear absorber can be obtained by attaching two horizontal springs in the configuration shown in Fig. 3, but for the sake of having more freedom in choosing the linear spring coefficient (for tuning purpose), horizontal springs are assumed to be initially un-stretched, i. e., $F_{s o}=0$.
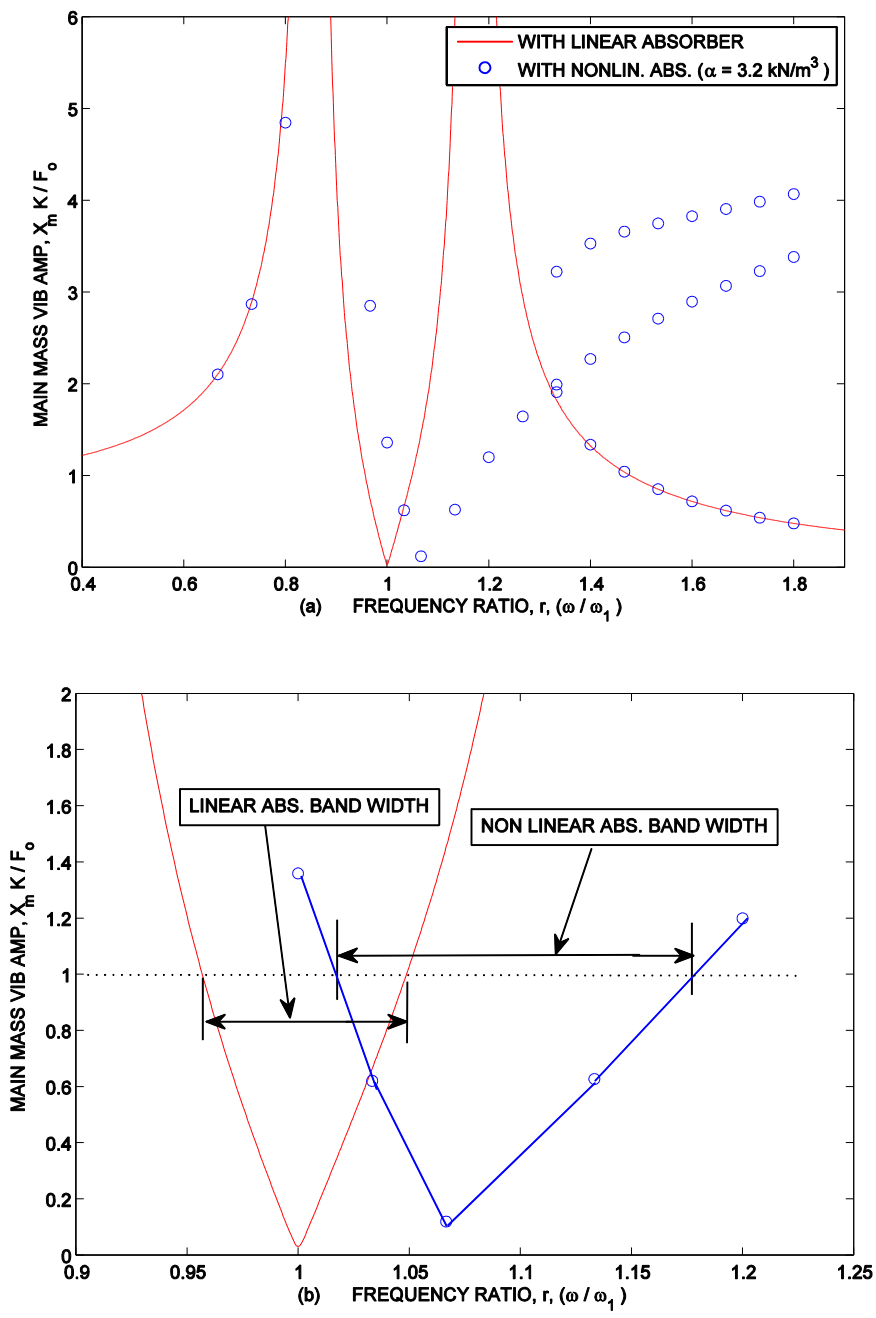

Fig. 5. Main mass normalized amplitude of vibration for different frequency ratios with linear and nonlinear absorbers 
If the horizontal springs are chosen with $k_{h}=200 \mathrm{~N} / \mathrm{m}$ and each with an un-stretched length of $25 \mathrm{~cm}$, then the nonlinear spring coefficient will be $\alpha_{a}=k_{h} / L^{2}=3.2 \mathrm{kN} / \mathrm{m}^{3}$. Fig. 5 shows the normalized amplitude of vibration of the main mass for different frequency ratios with linear and nonlinear absorbers. If the band width is defined as the frequency range at which the amplitude of vibration of the main mass is below its spring's static extension due to a static force of amplitude $F_{o}$, then it is clear that the band width is almost doubled when employing the nonlinear absorber (Fig. 5-b). The multivalued responses are also clear in Fig. 5-a, but one need not to worry about them since they are beyond the absorber's working range.

Fig. 6 shows the normalized steady state relative amplitude of vibration for the linear and the nonlinear absorbers during operation at different frequency ratios. It is clear that the nonlinear absorber amplitude of vibration is less than that of the linear absorber for the whole working ranges of the absorbers. This gives nonlinear absorber another advantage regarding an important design factor which is the available space limitations.

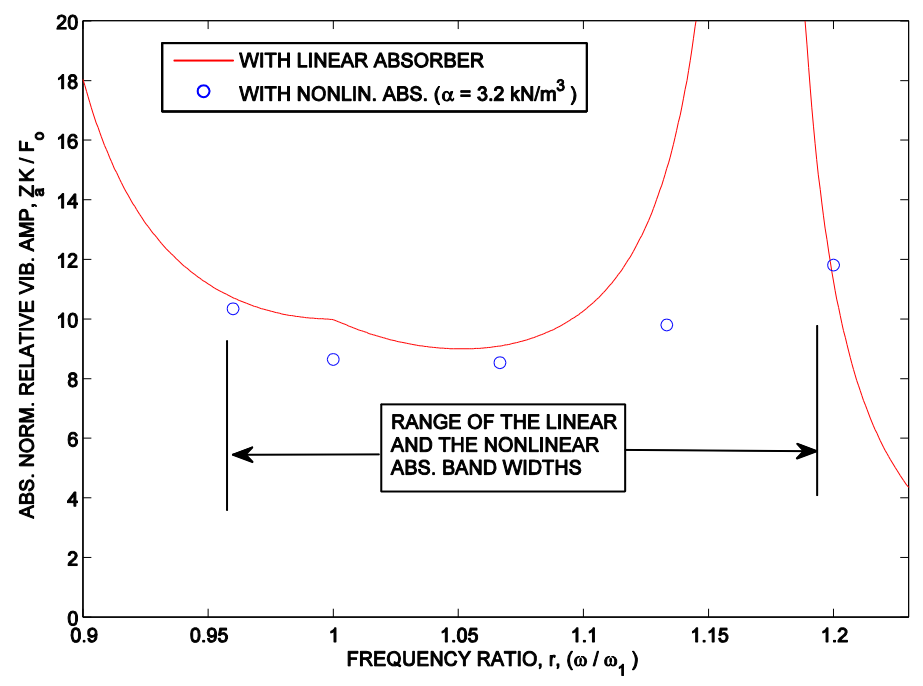

Fig. 6. Normalized relative amplitude of vibration for the linear and the nonlinear absorbers at different frequency ratios

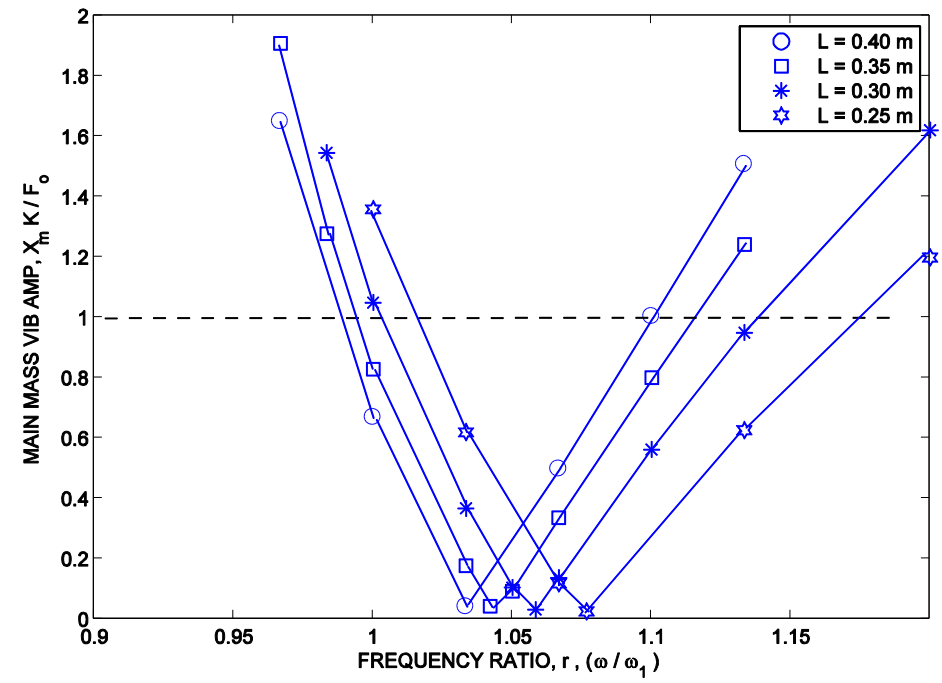

Fig. 7. Normalized relative amplitude of vibration for the linear and the nonlinear absorbers at different frequency ratios

Fig. 7 shows the band widths for different nonlinear absorbers obtained by changing the value of length of the added horizontal springs. It is clear that as the length is decreased, the absorber's effective band width increases. This is due to the increase in the nonlinearity in the absorber spring stiffness as the added spring length is decreased, see equation (8). Caution should however be taken here in order not to bring the undesirable nonlinear effects close to the absorber's effective band width. 


\section{Conclusions}

In this work, a system consisting of a main structure with a nonlinear tuned vibration absorber having a hardening spring attached to it is considered. The dynamics of a main structure, where the absorber is attached, and that of the absorber are analyzed using first order harmonic balancing method and the results are compared with that of a similar structure but with a linear tuned vibration absorber attached to it. The nonlinearity in the absorber spring is a cubic nonlinearity and is achieved by attaching two similar, relatively soft, linear springs to the absorber mass so that they are initially normal to the absorbers displacement axis. This simple configuration gave the required nonlinearity. The results of the analysis are in agreement with the known features of nonlinear absorbers. The absorber's effective frequency band width is increased by employing a nonlinear absorber instead of a linear one. The absorber's displacement amplitude during operation is decreased when using a nonlinear absorber. It is also found that the absorber's effective band width depends on the original length of the added springs in such a manner that it increases as the springs original length is decreased.

\section{References}

[1] H. Frahm, Device for Damping Vibrations of Bodies. U.S. Patent \#989958, 1911.

[2] J. P. Den Hartog, Mechanical vibrations 4th ed. (New York: Dover Publications, Inc., 1985).

[3] S. S. Patil, S. B. Javehri, and C.G. Konapure, Effectiveness of multible tuned mass dampers, International Journal of Engineering and Innovative Technology, 1, 2012, 78-83.

[4] T. Igusa,T. and K. Xu, Vibration control using multiple tuned mass damper, Journal of sound and vibration, 175, 1994, $491-503$.

[5] A. Kareem, and S. Kline, Performance of multiple mass dampers Under random loading, Journal of Structural Engineering, 121(2), 1995, 348-361.

[6] H. Yamaguchi, and N. Harnpornchai, Fundamental characteristics of multiple tuned mass dampers for suppressing harmonically forced oscillations, Earthquake Engineering \& Structural Dynamics, 22(1), 1993, 51-62.

[7] R. E. Roberson, Synthesis of a nonlinear dynamic vibration absorber, J. Franklin Inst., 254, 1952, 205-220.

[8] J. B.Hunt, and J. C. Nissen, The broadband dynamic vibration absorber, Journal of Sound and Vibration, 83(4), 1982, 573-578.

[9] J. C.Nissen, K. Popp, B. Schmalhorst, Optimization of a non-linear dynamic vibration absorber, Journal of Sound and Vibration, 99(1), 1985, 149-154.

[10] A. Soom, and M. Lee, Optimal design of linear and non-linear vibration absorbers for damped systems, Trans ASME Journal of Vibration and Acoustics, 105(1), 1983, 112-119.

[11] I. N. Jordanov, and B. I. Cheshankov, Optimal design of linear and non-linear dynamic vibration absorbers, Journal of Sound and Vibration, 123(1), 1988, 157-170.

[12] H. J. Rice, Combinational instability of the nonlinear vibration absorber, Journal of Sound and Vibrations, 108(4), 1986, 526-532.

[13] J. Shaw, S. W. Shaw, and A. G. Haddow, On the response of the nonlinear vibration absorber, Journal of Nonlinear Mechanics, 24, 1989, 281-293.

[14] M. Ghadimi and H.D. Kaliji, Application of the harmonic balance method on nonlinear equations, World Applied Sciences Journal, 22(4), 2013, 532-537.

[15] A. Beléndez, D.I. Méndez, T. Beléndez, A. Hernández, and M.L. Álvarez, Harmonic balance approaches to the nonlinear oscillators in which the restoring force is inversely proportional to the dependent variable, Journal of Sound and Vibration, 314(3-5), 2008, $775-782$.

[16] Y. S. Hsu, N. S. Ferguson, and M. J. Brennan, The experimental performance of a nonlinear dynamic vibration absorber, Proceedings of IMAC XXXI Conference \& Exposition on Structural Dynamics, Garden Grove, CA, USA, February 11-14, 2013. 